\title{
Control of Phase and Phase Slope with Reinvestigation of 1D CRLH Transmission Lines
}

\author{
Dongho Jeon $\cdot$ Bomson Lee
}

\begin{abstract}
The conventionally classified waves are reclassified in this paper, including the waves associated with metamaterial (MTM) transmission lines. Various types of guided waves in periodic structures are analyzed for 1D Composite Right/Left Handed (CRLH) transmission lines (TL). In addition, dispersion diagrams for 1D CRLH, RH, and LH TLs are presented in terms of radiation conditions. We have refined the design equations of the conventional 1D CRLH $\mathrm{TL}$ and proposed a condition to realize the desired phase shift and its slope per unit cell. This condition has been applied to the conventional Wilkinson power divider and realized into MTM added $90^{\circ}$ hybrid coupler. Based on the $10 \mathrm{~dB}$ return loss and the $90 \pm 10^{\circ}$ phase shift, the bandwidths of the MTM added $90^{\circ}$ hybrid coupler are $82.25 \%$ and $103.5 \%$ against the conventional $23 \%$ and $52.5 \%$, respectively.
\end{abstract}

Key words: Metamaterial, CRLH, Phase Shift, Phase Slope.

\section{Introduction}

There has been intense research conducted on metamaterial based transmission lines. A tremendous amount of structures and theories of 1D CRLH have been studied and analyzed [1] [6]. The conventional transmission lines, which support TEM(Transverse Electromagnetic) waves and follow the right hand rule, have been characterized by the distributed series inductance $L$ (given in unit of $\mathrm{H} / \mathrm{m}$ ) and shunt capacitance $C$ (given in unit of $\mathrm{F} / \mathrm{m}$ ). By adding a lumped series capacitance $C_{0}$ and shunt inductance $L_{0}$ periodically with a unit cell size of $d$ (much smaller than one wavelength), the CRLH is constructed and a number of applications have then been conducted [7] [12]. Usually, the lumped series capacitance $C_{0}$ is realized by transverse, inter digital cut or chip capacitor on the signal line, while the lumped shunt inductance is achieved by either shunt stub [13] line or chip inductor. In either instance of realizing lumped elements, some power will leak out of both the series and shunt cuts and the stub lines. These radiation losses are inevitable. Most applications, such as the phase shifter, power combiner, and power divider should carry the power to the load while minimizing the radiation losses. On the other hand, with some antenna applications such as leaky wave antenna, this radiation effect needs to be increased and even controlled if possible [14].

In this paper, the radiation condition and types of guided waves are provided with EM and circuit simulations. We have presented dispersion diagrams for $1 \mathrm{D}$ CR$\mathrm{LH}, \mathrm{RH}$, and LH transmission lines considering backfire, broadside, and endfire radiations. Furthermore, we have derived and provided the design equations for the 1D CRLH TL which has a desired phase shift and its slope per unit cell. The proposed methods are useful to widen the bandwidth of the phase shift between output ports. The phase shift of the conventional coupler is maintained only at the design frequency. However, the proposed method exhibits a significantly larger phase slope bandwidth. The effects of the closed-form equations are illustrated with a design example.

\section{II . Design Equations for Lossless 1D CRLH Line}

Fig. 1 shows the equivalent circuit of the 1D CRLH transmission line. For a lossless 1D CRLH TL(Fig. 1. $\left.R_{0}=G_{0}=0\right)$, the RH TL consists of distributed series capacitance $C(\mathrm{~F} / \mathrm{m})$ and distributed shunt inductance $L$ $(\mathrm{H} / \mathrm{m})$ and their product is given by

$$
L C=\mu \varepsilon_{0} \varepsilon_{e}
$$

Manuscript received October 10, 2012 ; Revised November 20, 2012 ; Accepted November 22, 2012. (ID No. 20121010-022J)

Dept. of Electronics and Radio Engineering, College of Electronics and Information, Kyung Hee University, Yongin, Korea. Corresponding Author : Bomson Lee (e-mail : bomson@khu.ac.kr)

This is an Open-Access article distributed under the terms of the Creative Commons Attribution Non-Commercial License (http:/creativecommons.org/licenses/ by-nc/3.0) which permits unrestricted non-commercial use, distribution, and reproduction in any medium, provided the original work is properly cited. 


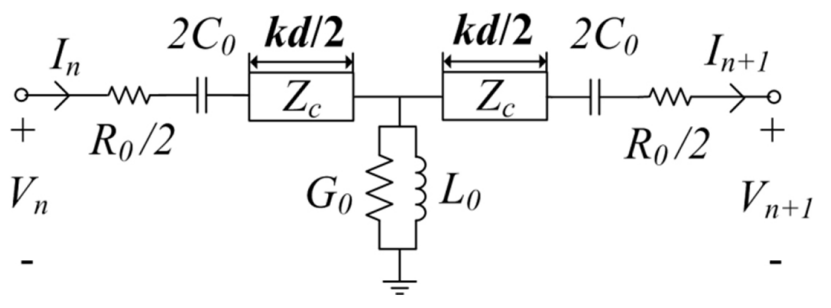

Fig. 1. Equivalent circuit of 1D CRLH transmission line.

where $\varepsilon_{0}$ and $\mu_{0}$ are the permittivity and permeability of the free space, respectively, and $\varepsilon_{0}$ is the relative effective dielectric constant. Most applications using a lossless CRLH TL are commonly based on the matched condition.

$$
Z_{C}=\sqrt{\frac{L}{C}}=\sqrt{\frac{L_{0}}{C_{0}}}
$$

where $Z_{C}$ is the characteristic impedance of the RH TL, $C_{0}$ is the series lumped capacitance and $L_{0}$ is the shunt lumped inductance. When the unit cell size, $d$, of the transmission line is much smaller than one wavelength, the phase shift per unit cell, $\phi(\omega)$, is given by

$$
\phi\left(\omega_{0}\right)=-\beta d \approx-\left(\omega_{0} \sqrt{L C} d-\frac{1}{\omega \sqrt{L_{0} C_{0}}}\right)=-k d+\frac{1}{\omega_{0} \sqrt{L_{0} C_{0}}}
$$

where $\beta$ is the propagation constant of the CRLH TL and $k$ is the propagation constant of the RH TL. When a specific phase shift $\phi\left(\omega_{0}\right)$ per unit cell is required at the center frequency of $\omega_{0}$, the design formulas for lumped elements, $C_{0}$ and $L_{0}$ can be expressed as

$$
C_{0}=\frac{1}{\omega_{0} Z_{C}\left[k d+\phi\left(\omega_{0}\right)\right]}
$$

and

$$
L_{0}=Z_{C}^{2} C_{0}
$$

The lumped capacitance $C_{0}$ can be realized by either a transverse cut or an inter-digital cut, and $L_{0}$ is commonly realized by a shunt shorted stub line or chip inductor. In the process of realizing lumped elements $L_{0}$ and $C_{0}$, some power is leaked out and the radiation rate, $\eta$ per unit cell due to a gap in the series and shunt line is the sum of each [3] and can be expressed as

$$
\eta=\eta_{\text {series }}+\eta_{\text {shunt }}=\frac{R_{0}}{Z_{C}}+\frac{G_{0}}{Y_{C}}
$$

Fig. 2 shows three particular radiation types as a function of frequency where $d$ is the size of a unit cell and $k_{0}$ is the wave number in free space. If the phase shift,

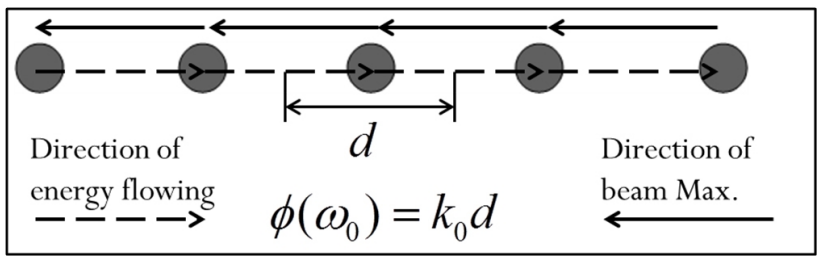

(a) Backfire radiation

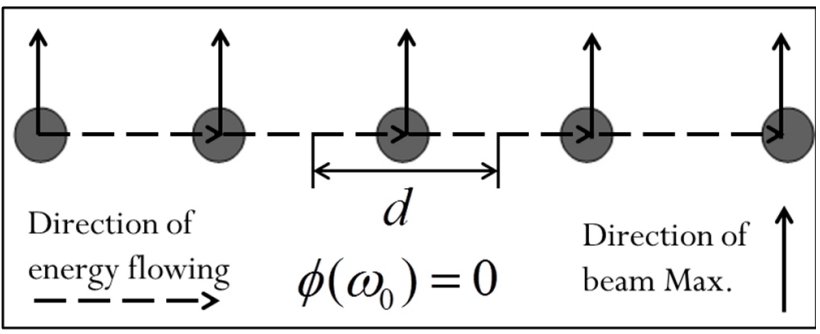

(b) Broadside radiation

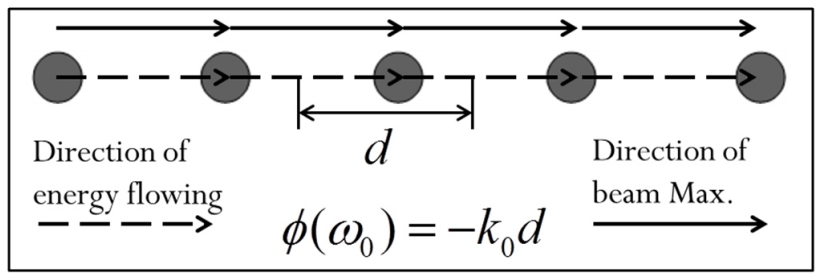

(c) Endfire radiation

Fig. 2. Radiation types in accordance with a specific phase shift $\phi\left(\omega_{0}\right)$.

$\phi\left(\omega_{0}\right)$ are $k_{0} d$ and $-k_{0} d$ at a specific frequency, radiation takes place toward the backfire and endfire direction, respectively, as seen in Fig. 2(a) and (c). When $\phi\left(\omega_{0}\right)$ is equal to zero $(\beta=0)$, the phase velocity along the line becomes infinite and the waves leaked out of the line due to the aforementioned discontinuities are directed toward the broadside as seen in Fig. 2(b). However, in either case of radiation the energy always flows to the right hand side. In other words, the group velocity is always positive. Since we have classified the radiated waves in Fig. 2 in the range of $\beta d$ from $-k_{0} d$ to $k_{0} d$, we now describe the wave behaviors when $\beta d>k_{0} d$. Fig. 3(a) shows the conceptual diagram for the transition between the endfire radiation and the RH TL. When the phase shift per unit cell, $\beta d$ is between $k_{0} d$ and $k d$ as seen in Fig. 3(a) the wave travels through the air near the TL, and this structure is constructed when a LH(Left handedness) characteristic is added on the RH TL. However, when the phase shift per unit cell, $\beta d$ is equal to the electrical length of RH TL, kd as seen in Fig. 3 (b) the wave tends to travel through the TL. Fig. 3(c) shows the conceptual diagram of a slow wave structure. Since the wave number in free space, $k_{0}$ needs to satisfy the equation.

$$
k_{0}^{2}=k_{z}^{2}+k_{x}^{2}
$$




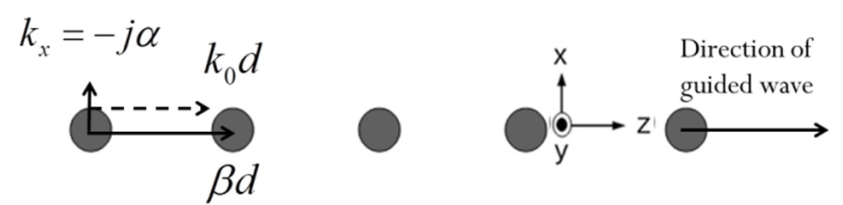

(a) Transition region from endfire radiation to $\mathrm{RH} \mathrm{TL}$

$$
\left(k_{0} d<\beta d<k d\right)
$$
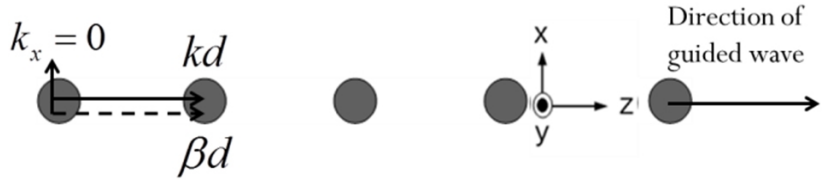

(b) $\mathrm{RH} \operatorname{TL}(\beta d=k d)$

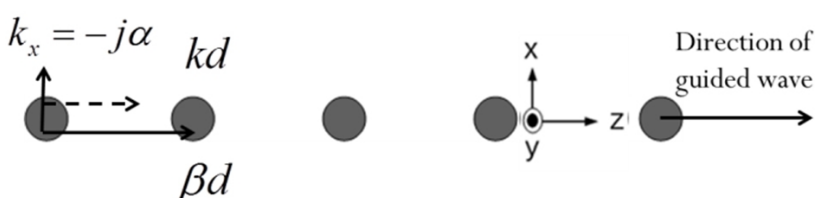

(c) Slow wave structure $(\beta d>k d)$

Fig. 3. The type of guided waves in array structures.

and $\beta d\left(k_{z} d\right)$ is greater than $k d$ for the slow wave structure, the normal component of the wave number $k_{x}$ must be $-j a$ (attenuating). The slow wave structure is usually constructed by periodically adding series inductance and shunt capacitance [5]. Fig. 4 shows the dispersion diagrams for the CRLH, RH, and LH TL. Fig. 4(a) shows the dispersion diagram for a pure RH and LH TL. The RH TL has a linear phase characteristic and is shown only in the positive region(the solid line on the right side of the diagram). The LH TL, on the other hand, has an exponentially increasing phase characteristic and is shown only in the negative region (the symbol line on the left side of the diagram). A typical dispersion diagram for a zero phase shift $(\beta d=0)$ 1D CRLH TL shown in Fig. 1 is depicted in Fig. 4(b), where the $k d$ is $30^{\circ}$ at $2 \mathrm{GHz}$. If the TL is designed to operate anywhere within the two light lines (dashed lines), radiation can occur. However, if the TL is designed to operate in the transition region between the light line and the $\mathrm{RH}$ $\mathrm{TL}$, the radiation doesn't take place in the far field region and only the evanescent wave occurs in the near region while it travels along the TL. Fig. 4(c) presents the case of a design in which radiation does not occur, where $k d$ is $30^{\circ}$ and $\phi\left(\omega_{0}\right)$ is $49^{\circ}$ at $2 \mathrm{GHz}$. If $\beta d$ is equal to $-\pi$, the incident wave will be totally reflected and this is called a Bragg's total reflection. This is because the reflected waves are all constructively added in phase.

\section{Analysis of the Condition for Realizing Desired Phase Shift and Phase Slope}

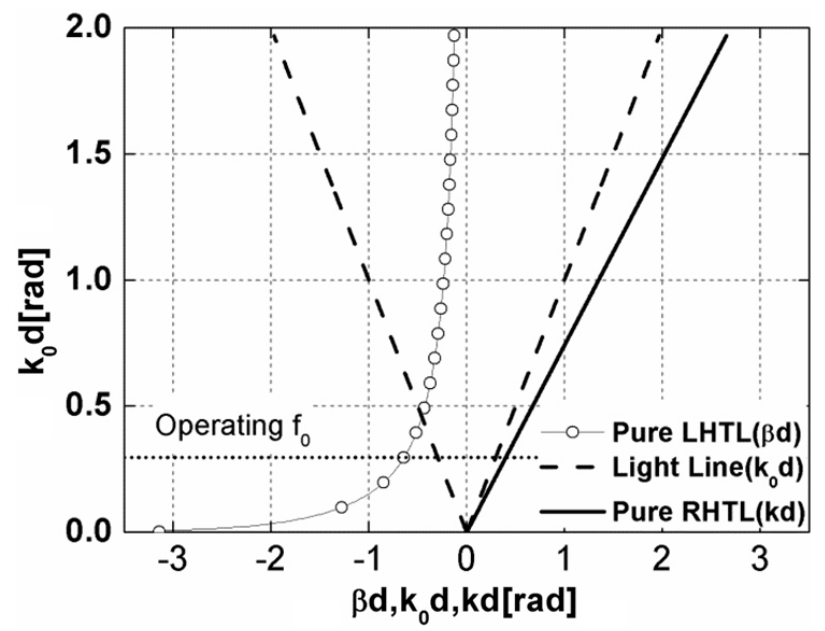

(a) Dispersion diagram for pure $\mathrm{RH}$ and $\mathrm{LH}$ TL

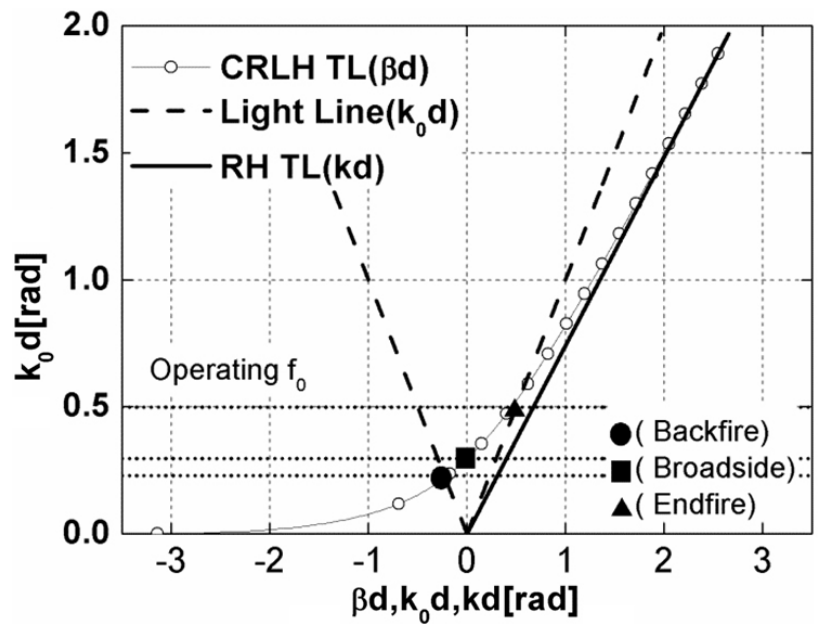

(b) Dispersion diagram for radiating 1D CRLH $\left(\phi\left(\omega_{0}\right)=0\right.$ at $2 \mathrm{GHz})$

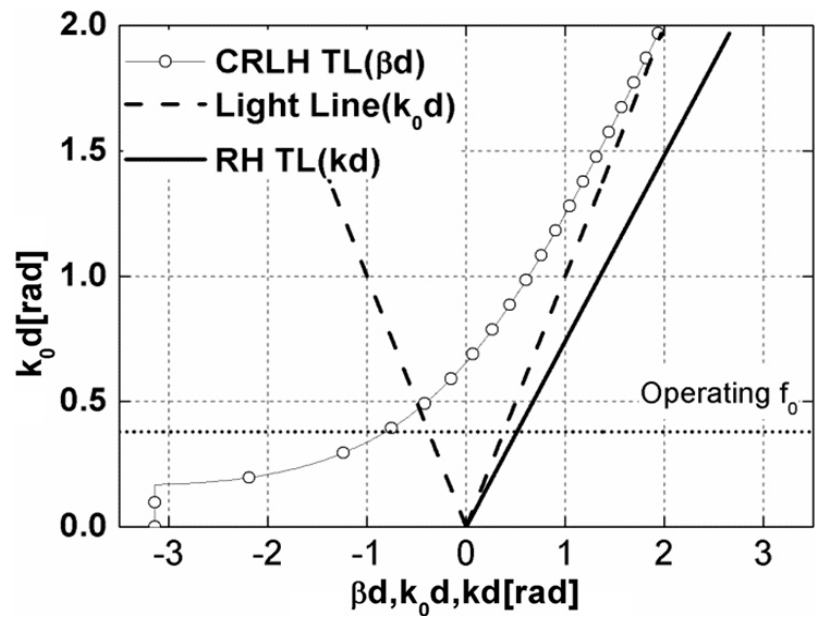

(c) Dispersion diagram for non-radiating 1D CRLH $\left(\phi\left(\omega_{0}\right)\right.$ $=49^{\circ}$ at $\left.2 \mathrm{GHz}\right)$

Fig. 4. Dispersion diagrams of $1 \mathrm{D} \mathrm{CRLH}, \mathrm{LH}$, and RH.

The conventional design equation for 1D CRLH TL is reinvestigated in this chapter. For a given $\mathrm{RH} T L$ 
characterized by $\mathrm{Z}_{\mathrm{C}}$ and $k d$, a specifically required phase shift $\phi\left(\omega_{0}\right)$ per unit cell is realized by loading $C_{0}$ and $L_{0}$ as given by (4) and (5). When a specific phase slope at $\omega_{0}$ is additionally required, we can still solve the problem by relaxing the condition for $Z_{C}$ and $k d$. The first step may be to take the derivative of Eq. (3) in Chapter II .

$$
\frac{d \phi}{d \omega_{0}}=\phi^{\prime}\left(\omega_{0}\right) \approx-\left(\sqrt{L C} d+\frac{1}{\omega_{0}^{2} \sqrt{L_{0} C_{0}}}\right) .
$$

By simultaneously solving the equations (3) and (8), the electrical length $k d$ of $\mathrm{RH}$ line becomes

$$
k d=-\frac{1}{2}\left[\phi\left(\omega_{0}\right)-\omega_{0} \phi^{\prime}\left(\omega_{0}\right)\right] .
$$

Then, the lumped capacitance $C_{0}$ and $L_{0}$ can be expressed as

$$
C_{0}=\frac{2}{Z_{C}} \cdot \frac{1}{\omega\left[\phi\left(\omega_{0}\right)-\omega \cdot \phi^{\prime}\left(\omega_{0}\right)\right]}
$$

and

$$
L_{0}=\frac{2 \cdot Z_{C}}{\omega_{0}\left[\phi\left(\omega_{0}\right)-\omega_{0} \cdot \phi^{\prime}\left(\omega_{0}\right)\right]} .
$$

Since the capacitance $C_{0}$, inductance $L_{0}$, and the electrical length $k d$ must have positive values, the following condition should be satisfied in the design process.

$$
\left|\phi\left(\omega_{0}\right)\right|<\omega_{0} \cdot \phi^{\prime}\left(\omega_{0}\right) .
$$

Fig. 5 shows the phase of $S_{21}$ for the five cases that have different phase slopes $\omega \cdot \phi^{\prime}(\omega)$ while the phase shift per unit cell $\phi(\omega)$ is fixed at zero-degree, as a function of frequency. When the $k d$ is $45^{\circ}\left(C_{0}=1.76 \mathrm{pF}\right.$, $L_{0}=4.41 \mathrm{nH}$ ), the slope of the $S_{21}$ is shown to be steeper. However, it shows the most gradual slope of $S_{21}$ when the $k d$ is $1^{\circ}\left(C_{0}=79.3 \mathrm{pF}, L_{0}=198.2 \mathrm{nH}\right)$ Fig. 6 shows the phase of $S_{21}$ when the phase slope $\omega_{0} \cdot \phi^{\prime}\left(\omega_{0}\right)$ is fixed with $-\pi / 2$ while the phase shift per unit cell varies from $-60^{\circ}\left(C_{0}=5.29 \mathrm{pF}, L_{0}=13.22 \mathrm{nH}, k d=75^{\circ}\right)$ to $60^{\circ}\left(C_{0}=1.06 \mathrm{pF}, L_{0}=2.64 \mathrm{nH}, k d=15^{\circ}\right)$. It is verified in Fig. 6 that the slope of $S_{21}$ for each unit cell can be maintained equally while the target phase shift is changing. The above results proved that any required phase shift and its slope can be achieved.

\section{Design of MTM added $90^{\circ}$ Hybrid Coupler Based on Conventional Wilkinson Power Divider}

The geometry of the MTM added $90^{\circ}$ hybrid coupler

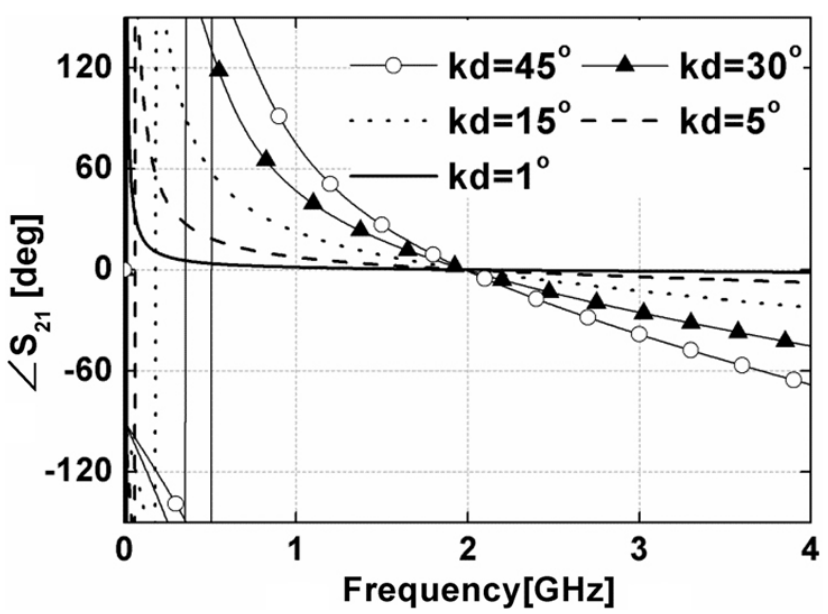

Fig. 5. Phase of $S_{21}$ for different $k d^{\prime}$ s $\left(\phi(\omega)=0^{\circ}\right.$ fixed $)$.

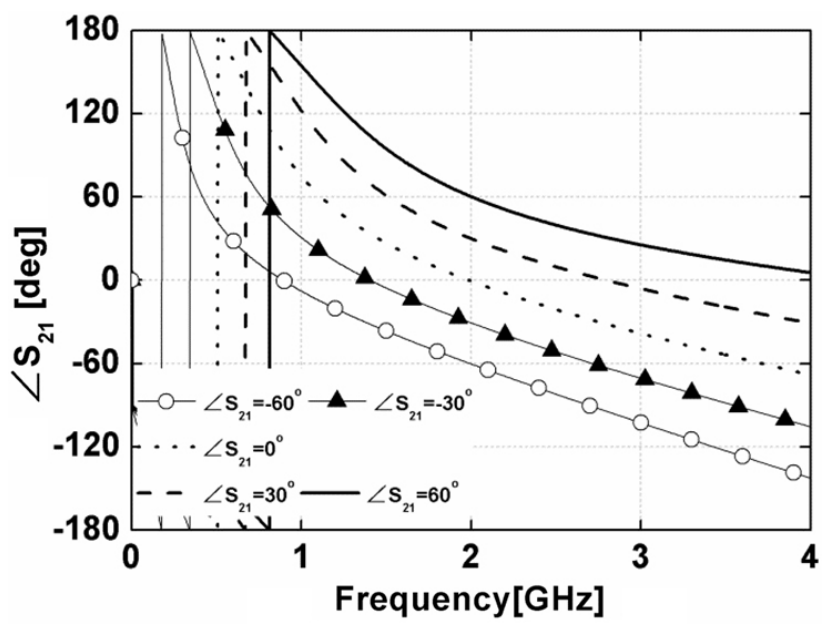

Fig. 6. Phase of $S_{21}$ with different phase shift at $\omega_{0}$ $\left(\omega_{0} \cdot \phi^{\prime}\left(\omega_{0}\right)=-\pi / 2\right.$ fixed $)$.

provided as an example is shown in Fig. 7. Each branch line consists of two MTM TLs with series chip capacitors and shunt inductors. This proposed structure can maintain good characteristics of the conventional Wilkinson power divider (Conv. W. power divider), such as equal power splitting between two output ports and achieving high isolations of the output ports as well as excellent return loss at all ports. While those advantages are maintained, $+45^{\circ}$ and $-45^{\circ}$ MTM phase shifting lines are inserted along the upper and lower branches, respectively. The design of the two MTM TLs is based on the theories in [4], [5]. The proposed structure is constructed on Teflon substrate with a height of $1.6 \mathrm{~mm}$ and relative permittivity of 2.17 . The width of the $50 \Omega$ RH microstrip TL is $5.3 \mathrm{~mm}$ and two $\lambda / 4$ branches are designed with a characteristic impedance of $\sqrt{2} Z_{0}(70.7 \Omega)$. The length $d$ of MTM TL for the upper branch is 12 $\mathrm{mm}$ and the lower branch is $25.2 \mathrm{~mm}$. Fig. 8 (a) shows 


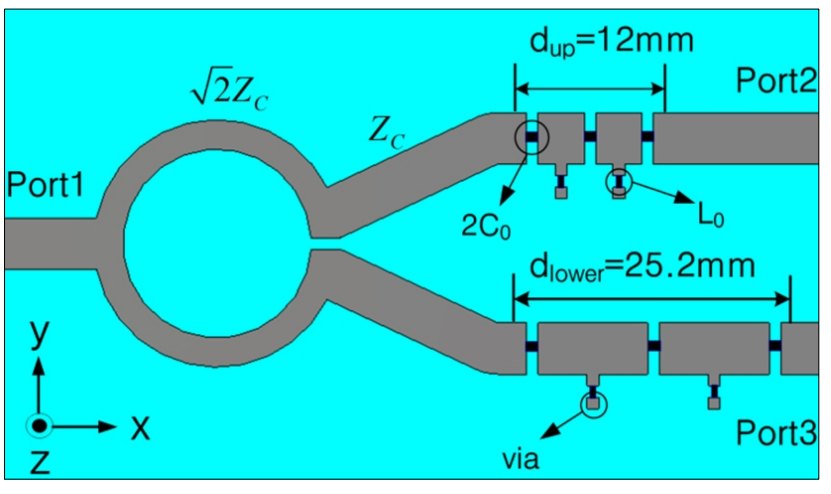

Fig. 7. Geometry of the MTM added $90^{\circ}$ hybrid coupler.

the circuit/EM simulated magnitude of $S_{11}, S_{21}$, and $S_{31}$ of a conventional $90^{\circ}$ hybrid coupler(Conv. $90^{\circ}$ hybrid) and the proposed MTM added $90^{\circ}$ hybrid coupler. Based on the $10 \mathrm{~dB}$ return loss, the bandwidth of the conventional $90^{\circ}$ hybrid coupler is shown to be 0.46 $\mathrm{GHz}(1.89 \sim 2.35 \mathrm{GHz})(23 \%)$, whereas that of the proposed MTM added coupler based on circuit/ EM simulations is $(2.54 \mathrm{GHz})(85.25 \%)$, respectively. Also, the return loss of the port 2 and port 3 are shown to be below the $10 \mathrm{~dB}$ over very large bandwidth. In Fig. 8(b), excellent isolation is shown between port 2 and 3. Based on the $10 \mathrm{~dB}$ return loss bandwidth, the bandwidth of $\left|S_{32}\right|$ for the conventional $90^{\circ}$ hybrid coupler is 0.8 $\mathrm{GHz}(01.63 \sim 2.43 \mathrm{GHz})(40 \%)$ and that of the MTM added coupler is $1.9 \mathrm{GHz}(0.87 \sim 2.77 \mathrm{GHz})(95 \%)$ which is almost same as that of the conventional Wilkinson power divider.

In Fig. 8(c), we show the phase shift of $S_{31}$ and $S_{21}$ for the conventional $90^{\circ}$ hybrid coupler and the proposed MTM added coupler (EM) as a function of frequency. Based on the $90 \pm 10^{\circ}$ phase shift, the bandwidth of the conventional $90^{\circ}$ hybrid coupler is $1.04 \mathrm{GHz}(1.71$ $\sim 2.75 \mathrm{GHz})(52.5 \%)$ while the proposed MTM added design is $2.07 \mathrm{GHz}(1.25 \sim 3.32 \mathrm{GHz})(103.5 \%)$.

\section{Conclusion}

Various types of radiated and guided waves have been examined founded on the conventional MTM TL theory. In addition, some useful closed-form solutions have been derived by solving equations of phase shift, phase slope, and matching condition. Based on the solutions, a novel MTM added $90^{\circ}$ hybrid coupler has been designed by placing $+45^{\circ}$ and $-45^{\circ}$ MTM TL sections at the ends of a conventional Wilkinson power divider. The performance of the proposed coupler has been greatly enhanced. Based on a $10 \mathrm{~dB}$ return loss, the bandwidth of the proposed coupler is $85.25 \%$ against the conventional $23 \%$. The bandwidth of the proposed cou-

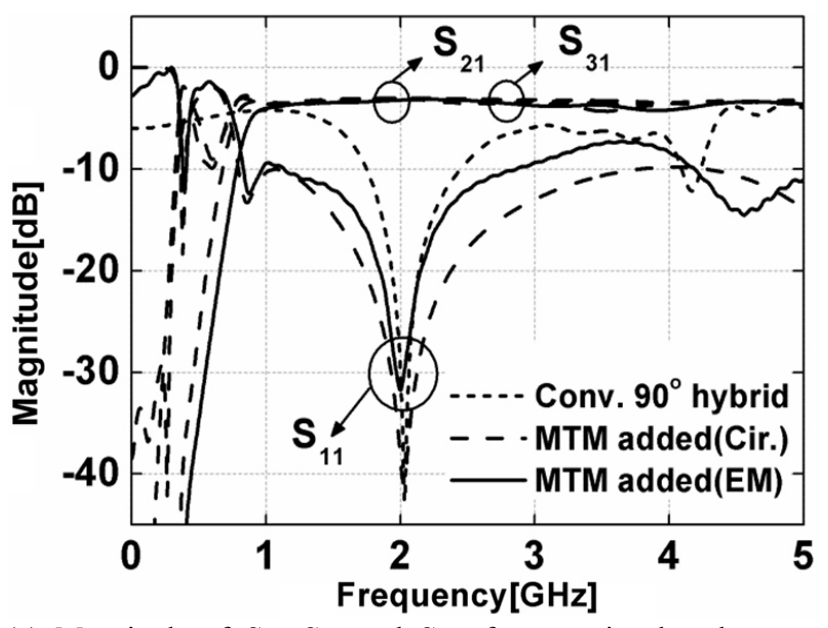

(a) Magnitude of $S_{11}, S_{21}$, and $S_{31}$ of conventional and proposed hybrid coupler

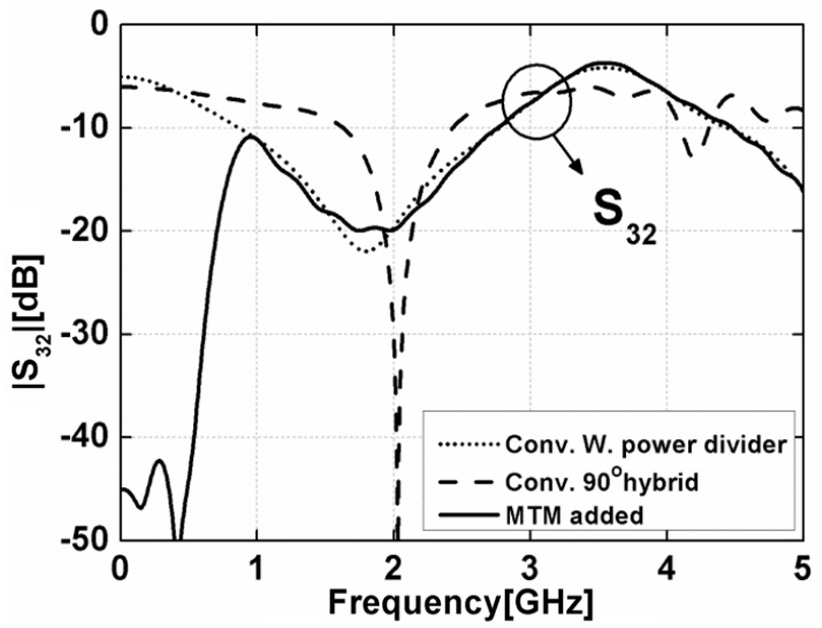

(b) EM-simulated magnitude of $S_{32}$

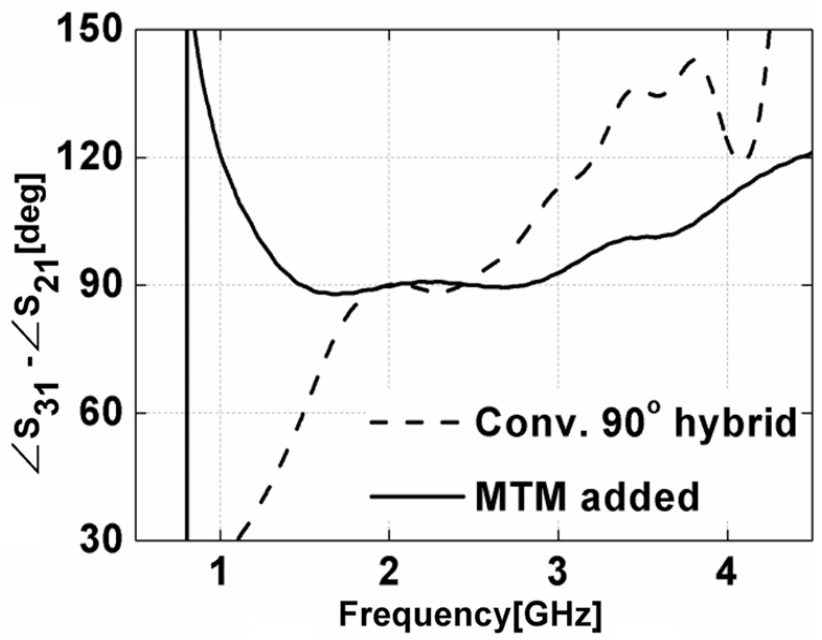

(c) EM-simulated phase shift between port 2 and 3

Fig. 8. EM/circuit simulation results.

pler based on $\mathrm{a} \pm 10^{\circ}$ phase shift between two output ports has been shown to be $103.5 \%$ against the conventional $52.5 \%$. 
This work was supported by the Mid-career Researcher Program through the National Research Foundation of Korea (NRF) grant funded by the Korea government (MEST) (No. 2012047938).

\section{References}

[1] G. V. Eleftheriades, A. K. Iyer, and P. C. Kremer, "Planar negative refractive index media using periodically L-C loaded transmission lines," IEEE Trans. Microwave Theory and Tech., vol. 50, no. 12, pp. 2702-2172, Dec. 2002.

[2] H. Okabe, C. Caloz, and T. Itoh, "A compact enhanced bandwidth hybrid ring using an artificial lumped-element left-handed transmission-line section," IEEE Trans. Microwave Theory Tech., vol. 52, no. 3, pp. 798-804, Mar. 2004.

[3] S. Lim, Christophe C. Caloz, and T. Itoh, "Metamaterial-based electronically controlled transmission-line structure as a novel leaky-wave antenna with tunable radiation angle and beam width," IEEE Trans. Microwave Theory Tech., vol. 53, no. 1, pp. 161173, Jan. 2005.

[4] C. Caloz, T. Itoh, "Transmission line approach of lefthanded (LH) materials and microstrip implementation of an artificial LH transmission line," IEEE Trans. Antennas and Propagation, vol. 52, no. 5, pp. 1159-1166, May 2004.

[5] S. Kahng, "Study on wave absorption of 1D-/2Dperiodic EBG structures and/or metamaterial layered media as frequency selective surfaces," Journal of The Korean Institute of Electromagnetic Engineering and Science, vol. 9, no. 1, pp. 46-52, Mar. 2009.

[6] H. Ahn, B. Kim, "Equivalent transmission-line sections for very high impedances and their application to branch-line hybrids with very weak coupling power," Journal of The Korean Institute of Electromagnetic Engineering and Science, vol. 9, no. 2, pp.
85-97, Jun. 2009.

[7] T. -G. Kim, B. Lee, "Metamaterial-based wideband rat-race hybrid coupler using wave lines," IET Microwaves, Antennas \& Propagation, vol. 4, no. 6, pp. 717-721, Jun. 2010.

[8] Y. Jung, B. Lee, "Design of compact and wideband metamaterial balun based on closed-form solutions," Microwave and Optical Technology Letters, vol. 52, no. 5, pp. 1153-1156, May 2010.

[9] M. A. Antoniades, G. V. Eleftheriades, "A broadband Wilkinson balun using microstrip metamaterial lines", IEEE Trans. Antennas \& Propagation, vol. 4, no. 5, pp. 209-212, 2005.

[10] H. Choi, T. Mun, Y. Jeong, J. Lim, S. Eom, and Y. Jung, "Dual-band group dealy circuit using $\lambda / 4$ composite right/left-handed short stubs," Journal of The Korean Institute of Electromagnetic Engineering and Science, vol. 11, no. 2, pp. 76-82, Jun. 2011.

[11] D. Kim, "A sub-wavelength focusing lens composed of a dual-plate metamaterial providing a negative refractive index," Journal of The Korean Institute of Electromagnetic Engineering and Science, vol. 12, no. 1, pp. 26-31, Mar. 2012.

[12] Y. Lee, H. Chung, and J. Choi, "Improving the isolation of MIMO antennas using split ring resonator," Journal of The Korean Institute of Electromagnetic Engineering and Science, vol. 10, no. 4, pp. 303-308, Dec. 2010.

[13] S. Jang, B. Lee, "Investigation of 1-D meta-structured leaky wave antennas using transmission line theory considering radiation effects," IEEE Antennas \& Propagation Society International Symposium, Jun. 2009.

[14] T. -G Kim, B. Lee, "Modeling and analysis of radiation effects for one-dimensional metamaterialbased transmission lines," IET, Microwaves, Antennas \& Propagation, vol. 4, issue 3, pp. 289-295, Mar. 2010. 
Dongho Jeon

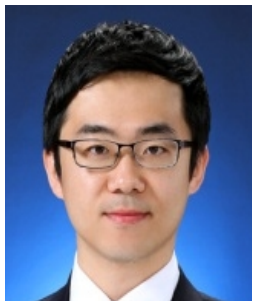

received a B.S. degree in Radio Communication Engineering from Kyung Hee University, Yong-in, Korea, in 2012. He is currently working toward a Master's degree in Electronics and Radio Engineering at Kyung Hee University. His fields of research include small antennas, passive devices, RFID reader antennas, wireless power transmission, and metamaterials.

\section{Bomson Lee}

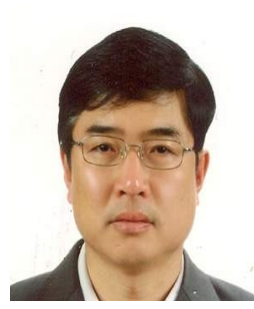

received a B.S. degree in Electrical Engineering from Seoul National University, Seoul, Korea, in 1982, and M.S. and $\mathrm{Ph} . \mathrm{D}$. degrees in Electrical Engineering from the University of Nebraska, Lincoln, NE, U.S.A., in 1991 and 1995, respectively. From 1982 to 1988 , He was with the Hyundai Engineering Company Ltd., Seoul, Korea. In 1995, he joined the faculty at Kyung Hee University, where he is currently a professor with the Department of Electronics and Radio Engineering. He was an editor-in-chief of the Journal of the Korean Institute of Electromagnetic Engineering and Science in 2010. He is an executive director (Project) in KIEES (Korea Institute of Electromagnetic Engineering \& Science). His research activities include microwave antennas, RF identification (RFID) tags, microwave passive devices, wireless power transmission and metamaterials. 